\title{
Gabriele Wesch-Klein
}

(Heidelberg)

DAS RÖMISCHE HEERWESEN: AUCH IM 21. JAHRHUNDERT

GEGENSTAND INTENSIVER FORSCHUNG

\section{Abstract}

The article gives a synoptic view of selected works to the most diverse parts of Roman military history.

\section{Key words}

Roman military history 
Die Römische Heeresgeschichte war ein Standbein des durch diese Gedenkschrift geehrten Althistorikers und Epigraphikers Géza Alföldy. Dies bezeugt eindrucksvoll sein gleichnamiger Sammelband ${ }^{1}$. Géza Alföldy prägte die Forschung zum Imperium Romanum in einigen Bereichen nachhaltig, insbesondere durch seine herausragenden Interpretationen von Inschriften, die unter anderem Angehörigen des römischen Heeres galten oder von diesen gesetzt wurden. Erinnert sei nur an seine Edition der Inschriften aus den Principia des Reiterkastells Aalen ${ }^{2}$. Besonders am Herzen lagen Géza Alföldy Fragen bezüglich der Religion des römischen Heeres, der Herkunft von Soldaten, Beförderungskriterien und die Karrieren von ritterlichen und senatorischen Offizieren. Im Zuge seiner Beschäftigung mit diesen Themen gelangte er immer wieder $\mathrm{zu}$ neuen, überraschenden Ergebnissen, die die Forschung bis heute nachhaltig beeinflussen und bisweilen zu kontroversen Diskussionen führten, wie etwa seine Überlegungen zur Vorverlegung des obergermanischen Limes auf die Linie Miltenberg - Welzheim unter dem Statthalter C. Popilius Carus Pedo ${ }^{3}$.

Das ungebrochene Interesse von Historikern, Archäologen und Laien an der römischen Militärgeschichte zeigen etwa unzählige an ein interessiertes breites Publikum gerichtete Schriften zu diesem Thema sowie die zahlreichen Tagungen, unter anderem die regelmäßig stattfindenden Limeskongresse. Im Jahr 2015 nahmen über 400 Personen aus 30 Nationen an dem von der Römisch-Germanischen Kommission und dem Bayrischen Landesamt für Denkmalpflege in Ingolstadt ausgerichteten 23. Limeskongress teil. Dort wurden rund 200 Vorträge gehalten.

Die allenthalben in der historischen Forschung in den vergangenen Jahren zu beobachtende Tendenz zu Abrissen, zu kurz und verständlich geschriebenen Einführungen und Überblicken macht auch vor der römischen Militärgeschichte nicht halt. Erwähnt seien exempli gratia aus der deutschsprachigen Fachliteratur Leonhard Burckhardts Werk „Militär-

\footnotetext{
${ }^{1}$ G. Alföldy, Römische Heeresgeschichte. Beiträge 1965-1985, Mavors Roman Army Researches 3, Amsterdam 1987.

${ }^{2}$ Die Inschriften aus den Principia des Alenkastells Aalen, mit einem Beitrag von V. HabichtWeinges, Fundberichte aus Baden-Württemberg 14, 1989, S. 293-338.

${ }^{3}$ Caius Popilius Carus Pedo und die Vorverlegung des obergermanischen Limes. Wiederabgedruckt und mit Nachträgen versehen, [in:] G. Alföldy, Römische Heeresgeschichte, S. 394-409; Erstpublikation: Fundberichte aus Baden-Württemberg 8, 1983, S. 55-67. Siehe dazu G. Alföldy, Die lineare Grenzziehung des vorderen Limes in Obergermanien und die Statthalterschaft des Gaius Popilius Carus Pedo, [in:] E. Schallmayer (Hg.), Limes Imperii Romani. Beiträge zum Fachkolloquium "Weltkulturerbe Limes” November 2001 in Lich-Arnsburg, Saalburg Schriften 6, Bad Homburg 2004, S. 7-20.
} 
geschichte der Antike", in dem der Autor auf 128 Seiten einen knappen Überblick über das Kriegswesen vom frühen Griechenland bis zum späten Rom bietet. Ebenfalls an interessierte Laien und Studierende richtet sich Christians Manns Buch „Militär und Kriegführung in der Antike”. Kurzgefasst und auf das Wichtigste beschränkt werden Bewaffnung, Kampftaktiken und Stellung der Soldaten in der Gesellschaft erläutert; in einem zweiten Teil geht Chr. Mann auf verschiedene Forschungsansätze ein. Eine kurzgefasste Einführung in Form eines chronologischen Überblicks über Krieg und Militärwesen von den Anfängen bis $476 \mathrm{n}$. Chr. bietet das von Jonathan P. Roth 2009 publizierte Buch „Roman Warfare”. Aus der Feder von Yann Le Bohec stammen in den vergangenen Dezennien zahlreiche Monographien zu Kriegen und Heerführung der Römer ${ }^{4}$. Seine 2017 veröffentlichte „Histoire des guerres romaines” bietet auf rund 590 Druckseiten einen detailreichen Überblick über die Kriege, die Rom vom 8. Jahrhundert v. Chr. bis $410 \mathrm{n}$. Chr. führte. Als nützlich und auch für Laien nachvollziehbar erachte ich Thomas Fischers reich bebilderte Ausführungen zu Tracht, Bewaffnung und Ausrüstung des Heeres, die 2014 unter dem Titel „Die Armee der Caesaren” erschienen sind. Marcus Junkelmanns an ein breiteres Publikum gerichtetes Buch „Die Legionen des Augustus” löste ein wahres Römerfieber aus. Römerfeste haben sich seitdem allenthalben etabliert und Groß und Klein zeigen eine ungebrochene Freude daran, sich in einen Römer, vorzugsweise einen römischen Soldaten, zu verwandeln. 29 Jahre nach der 1. Auflage steht seit 2015 die 15. gründlich überarbeitete und erweiterte Auflage dieses Buchs zur Verfügung, in die Marcus Junkelmann die neuesten Ergebnisse der experimentellen Archäologie einbezogen hat ${ }^{5}$ und sich kritisch mit manchen in den letzten Jahren geäußerten Ansichten auseinandersetzt, so etwa der Ansicht, Soldaten hätten Schläuche als tragbare Wasserbehälter benutzt ${ }^{6}$, was $M$. Junkelmann dezidiert negiert.

Auch die Aufarbeitung des komplexen Themas Militärgeschichte in Form von Handbüchern und Lexika prägt das Bild der Forschung im 21. Jahrhundert n. Chr. Zu erinnern ist vor allem an das von Yann Le Bohec und anderen 2015 herausgegebene dreibändige Werk „The Encyclopedia of the Roman Army”, sowie an den von Michael Alexander Speidel und Leonhard

\footnotetext{
${ }^{4}$ Siehe das beigefügte Literaturverzeichnis.

${ }^{5}$ Chr. Koepfer, F.W. Himmler, J. Löffl (Hg.), Die römische Armee im Experiment, Berlin 2011.

${ }^{6}$ M. Volken, The Water Bag of Roman Soldiers, JRA 21, 2008, S. 264-274.
} 
Burckhardt initiierten Supplementband zum Lexikon „Der Neue Pauly”, an dem zahlreiche Fachkollegen mitwirken; er soll 2018 veröffentlicht werden.

Eine Fortsetzung fanden die stets nützlichen Arbeiten über Geschichte einzelner Truppenkörper und ihre materielle Hinterlassenschaft. Erwähnt seien neben anderen die Untersuchungen von Hans Petrovitsch über die legio II Italica, die unter Marc Aurel und Lucius Verus aufgestellte norische „Hauslegion”, von Stephen J. Malone über die legio XX Valeria victrix sowie von Marcus Reuter zur legio XXX Ulpia victrix ${ }^{7}$. In Hinblick auf die Rangordnung des Heeres verdienen die eingehenden Untersuchungen von Olivier Richier und Patrice Faure unsere Beachtung ${ }^{8}$.

Am spannendsten sind für einen Historiker neue Quellen, zumal wenn sie eine Wissenserweiterung mit sich bringen oder sogar zum Umdenken zwingen. Für die militärgeschichtliche Forschung stellen die in den letzten Dezennien vermehrt zu Tage gekommenen Militärdiplome eine immense Bereicherung dar. Verwiesen sei dazu auf die zahlreichen Publikationen, die Werner Eck, Peter Weiß und andere vorgelegt haben'. Ihnen verdanken wir nicht nur ein Voranschreiten unseres Detailwissens, auch grundlegende Fragen, etwa die Erteilung des Eherechtes im Jahr 197 n. Chr. durch Septimius Severus, müssen anhand dieser Zeugnisse neu bewertet werden.

Eingehende Beachtung verdienen zwei Bronzeurkunden, die Peter Weiß ausführlich und konzise ediert sowie analysiert hat. Sie verifizieren Dezennien später die von Géza Alföldy 1967 aufgrund onomastischer Studien geäußerte Vermutung, Caracalla habe im Jahr 214 für seinen Orientfeldzug Thraker als Soldaten ausgehoben ${ }^{10}$. Bei den beiden Urkunden, den ersten ihrer Art, handelt es sich um instrumenta, die sich zwei Soldaten der beiden damals in Obergermanien stationierten Legionen erbaten, um die ihnen anlässlich ihrer ehrenhaften Entlassung im Dezember 240 n. Chr., erteilten Privilegien nachweisen zu können. Da Legionäre bis in die Spätantike keine Entlassungsurkunden zu erhalten pflegten, war mit solchen Urkunden kaum zu rechnen. Umso mehr stellt sich nun die Frage, was die

\footnotetext{
${ }^{7}$ St.J. Malone, Legio XX Valeria Victrix. Prosopography, Archaeology and History, BAR International Series 1491, Oxford 2006; M. Reuter, Legio XXX Ulpia Victrix. Ihre Geschichte, ihre Soldaten, ihre Denkmäler, Darmstadt-Mainz 2012.

${ }^{8}$ O. Richier, Centuriones ad Rhenum. Les centurions légionnaires des armées romaines du Rhin, Gallia Romana 6, Paris 2004; P. Faure, L'aigle et le cep. Les centurions légionnaires dans l'Empire des Sévères, Ausonius. Scripta Antiqua 54, 2, Bordeaux 2013.

${ }^{9}$ Eine Auswahl findet sich im Literaturverzeichnis.

${ }^{10} \mathrm{P}$. Weiß, Eine honesta missio im Sonderformat. Neuartige Bronzeurkunden für Veteranen der Legionen in Germania superior unter Gordian III. Chiron 45., 2015, S. 52.
} 
beiden Veteranen $\mathrm{zu}$ ihrer Bitte veranlasste und warum dieser stattgegeben wurde.

Bewegte Ende des 20. Jahrhunderts n. Chr. die Entdeckung der mutmaßlichen Relikte der Varus-Katastrophe die althistorische bzw. provinzialrömische Forschung in Deutschland, ist es heute das Schlachtfeld am Harzhorn (im heutigen Bundesland Niedersachsen), das seit seiner Entdeckung im Jahr 2008 Fachwelt und Laien fasziniert und eine Jahrzehnte lange, letztlich fruchtlose Diskussion um den Ort der Schlacht, die die Römer gegen Germanen unter Maximinus Thrax führten, beendet ${ }^{11}$. Es kann nun mehr als gesichert gelten, dass der römische Herrscher Maximinus Thrax (235-238 n. Chr.) wie von antiken Autoren tradiert, mit seinem Heer tief in germanisches Gebiet eindrang und dort Germanen besiegte ${ }^{12}$. Die präzise Kartierung der aufgefundenen Objekte unter Zuhilfenahme modernster wissenschaftlicher Methoden ermöglichte eine detaillierte Rekonstruktion der Kampfhandlungen. Die Objekte selber bieten einen willkommenen Einblick in Ausrüstung und Bewaffnung eines römischen Heers auf dem Marsch in den 30er Jahren des 3. Jahrhundert n. Chr. Von Bedeutung sind vor allem die aufgefundenen Geschoßspitzen, die sowohl Torsionsgeschützen als auch leichten Wurfspeeren zuzuordnen sind. Dabei konnte, und das ist von großer Wichtigkeit, nachgewiesen werden, dass Geschoßspitzen mit quadratischem Querschnitt der Spitze und Tülle unter 60 Gramm durchaus Katapultpfeilen zugeordnet werden können, was in der Forschung bislang kontrovers diskutiert wurde; entscheidend sind indes allein Größe und Gewicht $^{13}$. Da Geschoßspitzen dieser Art häufig in Limeskastellen vorkommen, bedeutet dies, dass über die Ausstattung dieser Kastelle mit Torsionsgeschützen unvoreingenommen nachgedacht werden muss, ebenso über den Einsatz dieser Schnellfeuerwaffen im Kampfgeschehen. Dies gilt auch für die Verwendung von Armbrüsten. Die in Xanten aufgefundenen Relikte einer frühkaiserzeitlichen manuballista ermöglichten neben einer eingehenden Analyse die Rekonstruktion und den Test einer Waffe, die einmal mehr den hohen technischen Stand der römischen Ausrüstung

11 Siehe etwa A. Lippold, Der Germanenfeldzug des Kaisers C. Julius Verus Maximinus im Jahre 235-36. Die „Historia Augusta“ und Rätien, Bayerische Vorgeschichtsblätter 49, 1984, S. 197-213 mit zahlreichen Verweisen auf die ältere Literatur.

${ }^{12}$ Von einer Schlacht mit den Germanen berichten vor allem Herodian 7,2 und HA, Max. 11, $7 \mathrm{ff}$.

${ }^{13}$ H. Pöppelmann, K. Deppmeyer, W.-D. Steinmetz (Hg.), Roms vergessener Feldzug. Die Schlacht am Harzhorn, Veröffentlichungen des Braunschweigischen Landesmuseums 115, Darmstadt 2013, bes. S. 275 f. 
zeigt $^{14}$. Die Studien von Erwin Schramm und Dietwulf Baatz zu römischen Geschützen erfahren somit eine willkommene Bereicherung ${ }^{15}$.

Welche überraschenden Erkenntnisse der archäologischen Militärforschung noch immer möglich sind, zeigt ein Blick auf den obergermanischen Limes. Galt bis vor Kurzem der Verlauf des Neckarlimes zwischen Heilbronn und Stuttgart als der klassische Flußlimes in Obergermanien während der 1. Hälfte des 2. Jahrhunderts, so konnte in den letzten Jahren eine vorgelagerte, deutlich östlich des Neckars verlaufende Wachtturm-Linie nachgewiesen werden ${ }^{16}$. Und auch eine Frage des vorderen Limes im 3. Jahrhundert n. Chr. ist wahrscheinlich gelöst: Palisadenreihe und Wall-Graben-System sind nicht gleichzeitig, sondern nacheinander, sich ablösend angelegt worden ${ }^{17}$. Selbstverständlich haben weiterhin unzählige archäologische Untersuchungen an vielen Anlagen sämtlicher römischer Limites in Europa, Afrika und in Asien zu wichtigen und weiterführenden Ergebnissen geführt, auf die hier nicht weiter eingegangen werden kann.

In Anbetracht der verheerenden Wunden, die Soldaten erleiden konnten, sei das Buch von Olaf Krause „Der Arzt und sein Instrumentarium in der römischen Legion" erwähnt, das eine gute und nützliche Zusammenstellung von Arztbestecken und Arztgräbern bietet, und somit eine willkommene Ergänzung zu den seit 1995 vorliegenden Studien von Juliane C. Wilmanns zum römischen Sanitätsdienst darstellt ${ }^{18}$.

Besonders beeindruckend ist das zweibändige, von mehreren Wissenschaftlern unter der Herausgeberschaft von Hélène Cuvigny 2011 publizierte Werk „Didymoi, une garnison romaine dans le désert oriental d'Égypte”, nicht zuletzt wegen der gut dokumentierten Befunde und außergewöhnlichen

\footnotetext{
${ }^{14}$ H.-J. Schalles (Hg.), Die frühkaiserzeitliche manuballista aus Xanten-Wardt, Xantener Berichte 18, Mainz 2010.

${ }^{15}$ E. Schramm, Die antiken Geschütze der Saalburg. Bemerkungen zu ihrer Rekonstruktion, Berlin 1918 (Nachdruck: Bad Homburg 1980 mit einer Einführung von D. Baatz, Beiheft zum Saalburg Jahrbuch); D. Baatz, Bauten und Katapulte des römischen Heeres, Mavors Roman Army Researches 11, Stuttgart 1994.

${ }^{16}$ St. Bender, Einem neuen Limes auf der Spur - Forschungen an der Nahtstelle von Odenwald- und Neckarlimes in Bad Friedrichshall, Archäologische Ausgrabungen in BadenWürttemberg 2011, S. 44-48; idem, Komplexes System von Sperranlagen am Neckarlimes bei Kochendorf, Archäologische Ausgrabungen in Baden-Württemberg 2014, S. 172-174.

${ }^{17}$ M.G. Meyer, K. Kortüm, Ein Jahrhunderträtsel (fast) gelöst - der neue Limesknick bei Welzheim, Archäologische Ausgrabungen in Baden-Württemberg 2015, S. 167-170.

${ }^{18}$ J.C. Wilmanns, Der Arzt in der römischen Armee der frühen und hohen Kaiserzeit, [in:] Ph.J. van der Eijk, H.F.J. Horstmanshoff, P.H. Schrijvers (Hg.), Ancient Medicine in its Sociocultural Context. Papers Read at the Congress Held at Leiden University 1992 (1995), Clio Medica 27, 1, S. 171-187; eadem, Der Sanitätsdienst im römischen Reich, Hildesheim 1995.
} 
Fundstücke mit teilweise hohem Seltenheitswert, so Reste von Textilien, die einst von Soldaten getragen wurden. Erwähnt seien die Überbleibsel eines cento, der als Kopfschutz unter dem Helm diente und die Reste einer Kappe in Helmform, die entweder als eigenständige Kopfbedeckung oder ebenfalls als Unterhelm Verwendung fand. Bleiben wir noch einen Augenblick auf dem Feld der materiellen Hinterlassenschaften. Die 1969 abgeschlossene Wiener Dissertation von Hannsjörg Ubl zu Waffen und Uniform des römischen Heeres war 44 Jahre lang nur schwer in Form von in einigen Bibliotheken eingestellten Kopien zugänglich. 2013 ist diese über weite Strecken noch immer grundlegende Arbeit im Druck erschienen, dankenswerterweise wurde neben der Druckseitenzählung die originale Paginierung beibehalten. Kai Michael Töpfers Dissertation "Signa militaria” schließt eine weitere Lücke. Unter anderem setzt sich K.M. Töpfer kritisch mit den in der älteren Literatur als originale Feldzeichenbestandteile bzw. Adler umlaufenden Objekte auseinander - mit ernüchternden Ergebnissen. So können alle Adlerplastiken, die als Feldzeichen angesprochen wurden, als solche ausgesondert werden. Ein weiteres Verdienst der Arbeit ist die Zuweisung der diversen signa an einzelne Truppenkörper und deren typologische Entwicklung, wobei sich Töpfer auf zahlreiche ikonographische Quellen stützt. Freilich bleibt weiteres zu tun. Wie er selber schreibt, ist etwa eine Untersuchung der Verwendung der Feldzeichen im Kampf und der Art der Signalübermittelung weiterhin ein Desiderat (S. 263). Erwähnung verdienen in diesem Zusammenhang Ernst Künzls Publikation zum Waffenschmuck ${ }^{19}$ sowie zwei fleißige Materialsammlungen: Erstens das 2010 erschienene Werk „Blasmusiker und Standartenträger im römischen Heer. Untersuchungen zur Benennung, Funktion und Ikonographie” von CristinaGeorgeta Alexandrescu und zweitens Stefan Zehetners Studie „Der Signifer. Stellung und Aufgaben in der kaiserzeitlichen Armee". Neben der ehrenvollen Pflicht, das Feldzeichen der Zenturie zu tragen und mit dem eigenen Leben zu schützen, kam den signiferi die Aufgabe zu, für die korrekte Abrechnung und Verwahrung des Soldes ihrer Kameraden Sorge zu tragen; die Grundlage für die fiskalischen Aufgaben des signifer bildet Zehetners Analyse der Zeilen 3 bis 10 des noch nicht komplett und abschließend edierten Hamburger Papyrus mit der Inventarnummer 445. Diese Quelle zeigt zugleich, dass es unter den signiferi schwarze Schafe gab, die in ihre eigene Tasche

${ }^{19}$ E. Künzl, Unter den goldenen Adlern. Der Waffenschmuck des römischen Imperiums, Regensburg-Mainz 2008. 
wirtschafteten ${ }^{20}$. Erfreulicherweise fand und findet derzeit das römische Heerwesen im 3. Jahrhundert und damit die Frage nach den Reformen der Severer und dem Beginn des Bewegungsheeres großes Interesse; leider ist die lesenswerte Dissertation von Ross H. Cowan zu diesem Thema nur schwer zugänglich $^{21}$. Die Krönung für einen siegreichen Feldherrn bildete ein Triumphzug in Rom. Studien zum römischen Triumph sind kein Desiderat, erinnert sei nur an die Arbeit von Ernst Künzl22. Ida Östenbergs 2009 publiziertes Buch „Staging the World” setzt neue Akzente. Sie beleuchtet den Triumphzug als ein riesiges Spektakel zur Erbauung der römischen Bevölkerung, das bestimmten Ritualen folgte. Ausführlich analysiert werden die im Zug zur Schau gestellten Objekte - von Waffen über Gefangene, Bildern mit Szenen aus dem Kriegsgeschehen bis hin zu lebenden exotischen Bäumen. Als erster soll Pompeius einen bis dahin in Rom unbekannten Baum mitgeführt haben; ein Beispiel, das Schule machte. Für I. Östenberg liegt der Sinn des Triumphes darin, Eroberungen, Siege und die Vorherrschaft Roms über die Oikumene und ihre Lebewesen den Zuschauern vor Augen zu führen, Rom als Herrscherin der Oikumene zu präsentieren.

Wenden wir uns einem anderen Sektor, nämlich juristischen Fragen, zu. Nachdem sich Jost H. Jung in den 80er Jahren des 20. Jahrhunderts ausführlich mit der rechtlichen Stellung römischer Soldaten befasst hatte ${ }^{23}$, wird dieses Thema in der neuesten Literatur wieder aufgegriffen, freilich ohne wirklichen Erkenntnisfortschritt. Dennoch bietet die von der rechtswissenschaftlichen Fakultät in Wien angenommene und 2012 unter dem Titel „Die rechtliche Stellung römischer Soldaten im Prinzipat” veröffentlichte Dissertation von Christian Schmetterer einen nützlichen und gut lesbaren Überblick ${ }^{24}$.

\footnotetext{
${ }^{20}$ St. Zehetner, Der Signifer. Stellung und Aufgaben in der kaiserzeitlichen Armee, Saarbrücken 2011.

${ }^{21}$ R.H. Cowan, Aspects of the Severan Field Army. The Praetorian Guard, Legio II Parthica and Legionary Vexillations, AD 193-238, Glasgow 2002.

${ }^{22}$ E. Künzl, Der römische Triumph. Siegesfeiern im antiken Rom, München 1988.

${ }^{23}$ J.H. Jung, Das Eherecht der römischen Soldaten, ANRW II 14, 1982, S. 302-346; idem, Die Rechtsstellung des römischen Soldaten. Ihre Entwicklung von den Anfängen Roms bis auf Diokletian, ANRW II 14, 1982, S. 963-1008.

${ }^{24}$ Siehe auch C. Kreuzaler, Zwischen Privilegierung und Diskriminierung. Die Sonderstellung der Soldaten im römischen Recht, [in:] B. Palme (Hg.), Die Legionäre des Kaisers. Soldatenleben im römischen Ägypten, Nilus, Studien zur Kultur Ägyptens und des Vorderen Orients 18, Wien 2011, S. 27-40; J. Meyer-Hermann, Testamentum militis - das römische Recht des Soldatentestaments. Entwicklung von den Anfängen bis zu Justinian, Aachen 2012.
} 
Werfen wir noch einen Blick auf die klassische Philologie. Eine Warnung hinsichtlich der Glaubwürdigkeit antiker Autoren beinhaltet Stefan Gerlingers Monographie zur römischen Schlachtenrhetorik ${ }^{25}$. Der Autor widmet sich den Erzählstrukturen bei Caesar, Sallust und Tacitus mit dem Ergebnis, dass diese in wesentlichen Punkten übereinstimmen und damit dem Leser bzw. Hörer wohl vertraut sind. Diese Schlachtenrhetorik bedient sich eines auf althergebrachten, oft in der Mythenwelt verankerten exempla gründenden Stils, „der in formelhaften Beschreibungen sowie Übertreibungen geübt ist und der durch rhetorische Bildung gesichert wird" (S. 366). Es geht also darum, beim Leser eine entsprechende dramatische oder atmosphärische Wirkung zu erzielen. Zudem können durch den geschickten Einsatz der Stilmittel für die Römer nachteilige Situationen ins Positive verkehrt werden. Die Absicht der antiken Autoren ist es also nicht, Schlachtenberichte abzufassen, sondern die Tugenden von Feldherren und Soldaten herauszuarbeiten, um so die Größe der militärischen Leistungen der Römer in ein positives Licht zu rücken und ihre Handlungsweise gegenüber Gegnern und Besiegten als edel und gerecht erscheinen zu lassen.

Die erwähnten Werke stellen einen Bruchteil der seit dem Jahr 2000 erschienenen Arbeiten zur römischen Heeresgeschichte dar; weitere sind dem beigefügten Literaturverzeichnis zu entnehmen, das wiederum lediglich eine Auswahl zu bieten vermag. Géza Alföldy hat streng genommen keine wirklich neuen Themen der Heeresgeschichte angegangen, denn die von ihm aufgegriffenen Themen waren bereits in der Forschung präsent, erinnert sei etwa an die Arbeiten von Alfred von Domaszewski, Konrad Kraft, Emil Ritterling, Arthur und Ernst Stein. Géza Alföldy hat es freilich verstanden, diese Themenbereiche nachhaltig voranzubringen, ihnen neuen Atem einzuflößen und vor allem Inschriften nach dem Motto hic saxa loquuntur als eine der wichtigsten Quellen der Heeresgeschichte $\mathrm{zu}$ etablieren und damit die Forschung entscheidend zu prägen. Soldaten und Offizieresindin seinen Aufsätzen zu Individuen geworden, die uns partiellen Einblick in ihr Leben, ihre Laufbahnen, ihre Familienverhältnisse und ihre Religiosität gewähren. Es bleibt zu wünschen, dass die Forschung des noch jungen 21 . Jahrhunderts in der Zukunft durch neues Gedankengut und progressive Forschungsansätze weitere Bereicherung erfahren darf.

${ }^{25}$ St. Gerlinger, Römische Schlachtenrhetorik. Unglaubwürdige Elemente in Schlachtendarstellungen, speziell bei Caesar, Sallust und Tacitus, Heidelberg 2008. 


\section{THE ROMAN MILITARY: A CONTINUING SUBJECT OF INTENSIVE SCIENTIFIC INQUIRY IN THE 21ST CENTURY}

\section{Summary}

History of the Roman army - in a broad sense - was one of the pillars of Géza Alföldy's scholarly studies and investigations. His inspiring interpretations of various inscriptions had a fundamental impact on the shape of contemporary science. He was particularly interested in such issues as religion of the Roman military, the origins of soldiers, the rules of promotion and the careers of officers with senatorial and equestrian lineage.

The article offers an overview of major studies in history of the Roman army published in the recent years. There is no doubt that many had been inspired by the work and writings of Géza Alföldy. Although it is merely a subjective selection, the list of publications appended to the article attests to the intensity of such research, showing that it truly flourishes.

Strictly speaking, Géza Alföldy did not come up with any novel research problems as far as the Roman military was concerned. He would address those which had been present in science, as it is documented by works of such scholars as Alfred von Domaszewski, Konrad Kraft, Emil Ritterling, Eric Birley or Arthur and Ernst Stein. Nonetheless, Géza Alföldy understood the essence and appreciated the need to pursue those studies in greater depth while utilizing epigraphy to the broadest possible extent. Quite rightly, he considered inscriptions to be the foremost sources in that respect. Guided by hic saxa loquntur as his motto, Alföldy determined the shape of methodology employed in studies of the Roman army through his work. The ordinary soldiers and officers became genuine individuals, which in a sense enabled one to gain insight into their personal lives, professional careers, family ties, and religious beliefs. Thus far, research in the two first decades of the $21^{\text {st }}$ century follows the same path. It may only be desired that, as a result, the current state of our knowledge will be substantially enriched.

\section{Ausgewählte neuere Bibliographie (ab Erscheiungsjahr 2000)}

Alföldy G., Dobson B., Eck W. (Hg.), Kaiser, Heer und Gesellschaft in der römischen Kaiserzeit. Gedenkschrift für Eric Birley, HABES 31, Stuttgart 2000.

Alexandrescu C.-G., Blasmusiker und Standartenträger im römischen Heer. Untersuchungen zur Benennung, Funktion und Ikonographie, Imagines 1, Cluj-Napoca 2010.

Allison P.M., People and Spaces in Roman Military Bases, Cambridge 2013.

Barth H.-L., Das Verhältnis des frühen Christentums zum Militär, [in:] W. Blümer, R. Henke M. Mülke (Hg.), Alvarium. Festschrift für Christian Gnilka, Jahrbuch für Antike und Christentum, Ergänzungsband 33, Münster 2002. 
Bidwell P., Hodgson N., The Roman Army in Northern England. XXI ${ }^{\text {st }}$ International Limes Congress 2009 which is meeting in Newcastle, Newcastle upon Tyne 2009.

Birley A.R., Garrison Life at Vindolanda. A Band of Brothers, Stroud 2002.

Bishop M.C., Coulston J.C. (Hg.), Roman Military Equipment. From the Punic Wars to the Fall of Rome, 2. Aufl. Oxford 2006.

Bishop M.C., Lorica Segmentata. I: A Handbook to Articulated Roman Plate Armour, JRMES Monograph No. 1, Braemar, Kirkgate u.a. 2002.

Bishop M.C., Handbook to Roman Legionary Fortresses, Barnsley 2012.

Borhy L., Zsidi P. (Hg.), Die norisch-pannonischen Städte und das römische Heer im Lichte der neuesten archäologischen Forschungen. 2. Internationale Konferenz über norischpannonische Städte, Budapest-Aquincum, 11.-14. September 2002, Budapest 2005.

Beutler F., Gugl, Chr. u.a. (Hg.), Der Adler Roms. Carnuntum und die Armee der Cäsaren, Mainz 2017.

Brandl U. (Hg.), Frauen und römisches Militär. Beiträge eines Runden Tisches in Xanten vom 7. bis 9. Juli 2005, BAR Int. Ser. 1759, Oxford 2008.

Breeze D.J., The Roman Army, London 2016.

Brice L.L., Holding a Wolf by the Ears. Mutiny and Unrest in the Roman Military, 44 B.C.-A.D., Thesis (Ph.D.) University of North Carolina 2003.

Brewer R.J. (Hg.), Roman Fortresses and their Legions. Papers in Honour of George C. Boon, London 2000.

Burckhardt L., Militärgeschichte der Antike. Beck Wissen, München 2008.

Busch A.W., Militär in Rom. Militärische und paramilitärische Einheiten im kaiserzeitlichen Stadtbild, Palilia 20, Wiesbaden 2012.

Busch A.W., Militär im severischen Rom. Bärtige Barbaren?, [in:] N. Sojc, A. Winterling, U. Wulf-Rheidt (Hg.), Palast und Stadt im severischen Rom, Stuttgart 2013, S. 105-122.

Clark J.H., Triumph in Defeat, Military Loss and the Roman Republic, Oxford 2014.

Collins R., Symmonds M.F.A., Weber M. (Hg.), Roman Military Architecture on the Frontiers Armies and their Architecture in Late Antiquity, Oxford 2015.

Coudry M., Humm M. (Hg.), Praeda. Butin de guerre et société dans la Rome républicaine. Kriegsbeute und Gesellschaft im republikanischen Rom, Stuttgart 2009.

Coulston J.C., "Armed and belted men". The Soldiery in Imperial Rome, [in:] J.C. Coulston H. Dodge (Hg.), Ancient Rome. The Archaeology of the Eternal City, Oxford 2000, S. 76-118.

Coulston J.C., All the Emperor's Men. Roman Soldiers and Barbarians on Trajan's Column, Oxford 2017.

Cowan R.H., Aspects of the Severan Field Army. The Praetorian Guard, Legio II Parthica and Legionary Vexillations, AD 193-238, Glasgow 2002.

Cowan R.H., Roman Conquests: Italy, Barnsley 2009.

Cuvigny H. (Hg.), Didymoi, une garnison romaine dans le désert Oriental d’Égypte. Praesidia du désert de Bérénice IV; 1: Les fouilles et le matériel, 2: Les textes. Institut français d'archéologie orientale, Fouilles de l'Ifao 64 und 67, Kairo 2011-2012.

Dąbrowa E. (Hg.), Roman Military Studies, Kraków 2001.

Dąbrowa E. (Hg.), The Roman Near East and Armenia, Kraków 2003.

Daly G., Cannae. The Experience of Battle in the Second Punic War, London u.a. 2002.

D’Amato R., Sumner G., Arms and Armour of the Imperial Roman Soldier. From Marius to Commodus, 112 BC-AD 192, London 2009.

Dando-Collins St., Caesar's Legion: The Epic Saga of Julius Caesar's Elite Tenth Legion and the Armies of Rome, New York 2002.

Dando-Collins St., Legions of Rome: The Definitive History of Every Imperial Roman Legion, London 2010. 
De Blois L., Lo Cascio E., The Impact of the Roman Army (200 BC-AD 476). Economic, Social, Political, Religious and Cultural Aspects. Proceedings of the Sixth Workshop of the International Network Impact of Empire, Leiden 2007.

Depeyrot G., Les légions face aux Barbares. La colonne de Marc Aurèle, Paris 2011.

Dillon S., Welch, K.E. (Hg.), Representations of War in Ancient Rome, Cambridge u.a. 2006.

Dobson M., The Army of the Roman Republic. The Second Century BC, Polybius and the Camps at Numantia, Spain, Oxford 2008.

Dolata J., Römische Ziegelstempel aus Mainz. Teil 1: Militärische Ziegelstempel des 1. Jahrhunderts (Materialvorlage), Mainzer Archäologische Schriften 13, Mainz 2014.

Dreier Chr., Römisches Militär in Riegel im 1. Jahrhundert n. Chr., [in:] C. Bücker (Hg.), Regio archaeologica, Archäologie und Geschichte an Ober- und Hochrhein. Festschrift für Gerhard Fingerlin zum 65. Geburtstag, Rahden, Westf. 2002, S. 27-39.

Drogula F.K., Commanders and Command in the Roman Republic and Early Empire, Chapel Hill, NC 2015.

Eck W., Pangerl A., Moesia und seine Truppen. Neue Diplome für Moesia und Moesia superior, Chiron 38, 2008, S. 317-387.

Eck W., Pangerl A., Weiß P., Gesamtindex zu den Publikationen der neuen Diplome von Moesia, Moesia superior und Moesia inferior, Chiron 38, 2008, S. 389-394.

Eck W., Kommunikation durch Herrschaftszeichen. Römisches Militär und römische Amtsträger in den Provinzen, [in:] O. Hekster (Hg.), Ritual Dynamics and Religious Change in the Roman Empire, Leiden 2009, S. 213-237.

Eck W., Rekrutierung für das Römische Heer in den Provinzen Kleinasiens. Das Zeugnis der Militärdiplome, [in:] O. Tekin (Hg.), Ancient History, Numismatics and Epigraphy in the Mediterranean World, Istanbul 2009, S. 137-142.

Eck W., Septimius Severus und die Soldaten. Das Problem der Soldatenehe und ein neues Militärdiplom, [in:] B. Onken, D. Rohde (Hg.), In omni historia curiosus. Studien zur Geschichte von der Antike bis zur Neuzeit. Festschrift für Helmuth Schneider zum 65. Geburtstag, Philippika 47, Wiesbaden 2011, S. 63-77.

Eck W., Diplomata militaria für Prätorianer, vor und seit Septimius Severus. Eine Bestandsaufnahme und ein Erklärungsversuch, Athenaeum 100, 2012, S. 321-336.

Eck W., Die Seuche unter Mark Aurel. Ihre Auswirkungen auf das Heer, [in:] E. Lo Cascio (Hg.), L'impatto della „Peste antonina”, Bari 2012, S. 63-78.

Eck W., Das Römische Heer und die kleinasiatischen Provinzen während der Hohen Kaiserzeit, [in:] Fischer, J., Der Beitrag Kleinasiens zur Kultur- und Geistesgeschichte der griechisch-römischen Antike. Akten des internationalen Kolloquiums Wien, 3.-5. November 2010, Wien 2014, S. 87-98.

Eck W., Pangerl A., Neue Diplome für die Truppen von Moesia superior und inferior, ZPE 192, 2014, S. 215-237.

Eck W., Pangerl A., Das vierte Diplom für die Provinz Galatia et Cappadocia, ausgestellt im Jahr 99, ZPE 192, 2014, S. 238-246.

Eck W., Pangerl A., Weiß, P., Edikt Hadrians für Prätorianer mit unsicherem römischen Bürgerrecht, ZPE 189, 2014, S. 241-253.

Eck W., Pangerl A., Die Konstitution des Commodus für das Heer von Pannonia inferior, publiziert im August des Jahres 193, [in:] S. Panzram, W. Riess, Chr. Schäfer (Hg.), Menschen und Orte der Antike. Festschrift für Helmut Halfmann zum 65. Geburtstag, Pharos, Studien zur griechische-römischen Antike 34, Rahden/Westf. 2015, S. 281-286.

Eich A., Der Wechsel zu einer neuen grand strategy unter Augustus und seine langfristigen Folgen, Historische Zeitschrift 288, 2009, S. 561-611.

Eich A., Die Verwaltung der kaiserzeitlichen römischen Armee. Studien für Hartmut Wolff, Historia Einzelschriften 211, Stuttgart 2010.

Eich A., Die römische Kaiserzeit. Die Legionen und das Imperium, München 2014.

Erdkamp P. (Hg.), A Companion to the Roman Army, Oxford 2007. 
Faure P., L'aigle et le cep. Les centurions légionnaires dans l'Empire des Sévères, Ausonius. Scripta Antiqua 54, 2 Bände, Bordeaux 2013.

Fields N., The Roman Army. The Civil Wars 88-31 B.C., Oxford 2008.

Fischer Th., Die Armee der Caesaren. Archäologie und Geschichte, 2. überarbeitete und aktualisierte Aufl., Regensburg 2014.

Flügel C., Obmann J. (Hg.), Römische Wehrbauten. Befund und Rekonstruktion. Kolloquiumsband: Arbeitsgespräch excelsae turres quater divisae am 5. Juli 2010, München 2013.

Fuhrmann C.J., Policing the Roman Empire. Soldiers, Administration, and Public Order, New York-Oxford 2012.

Gerlinger St., Römische Schlachtenrhetorik. Unglaubwürdige Elemente in Schlachtendarstellungen, speziell bei Caesar, Sallust und Tacitus, Heidelberg 2008.

Gilbert F., Le soldat romain à la fin de la République et sous le Haut-Empire, Paris 2012.

Giles R.D., Roman Soldiers and the Roman Army. A Study of Military Life from Archaeological Remains, 6. Aufl. Oxford 2012.

Goldworthy A., The Complete Roman Army, London 2003.

Günther R., Der Krieg - Sache der Männer?, [in:] E. Hartmann, U. Hartmann, K. Pietzner (Hg.), Geschlechterdefinitionen und Geschlechtergrenzen in der Antike, Stuttgart 2007, S. 87-98.

Handy M., Die Severer und das Heer, Studien zur Geschichte 10, Berlin 2009.

Hanson W.S. (Hg.), The Army and Frontiers of Rome. Papers offered to D.J. Breeze on the Occasion of his Sixty-fifth Birthday and his Retirement from Historic Scotland, JRA Suppl. Ser. 74, Portsmouth 2009.

Herz P., Schmid, P., Stoll, O. (Hg.), Handel, Kultur und Militär. Die Wirtschaft des AlpenDonau-Adria-Raumes, Berlin 2011.

Horvat J., The Roman Army between the Alps and the Adriatic, Opera Instituti Archaeologici Sloveniae 31. Studia Alpium et Adriae I, Ljubljana 2016.

Hoyos B.D. (Hg.), A Companion to the Punic Wars, Malden, MA 2011.

Jiménez de Furundarena A., Cohors I Lucensium Equitata, Hispania Antiqua 40, 2016, 169-190.

Junkelmann M., Die Legionen des Augustus. 15. gründlich überarbeitete und erweiterte Auflage, München 2015.

Keaveney A., The Army in the Roman Revolution, London-New York 2007.

Kemkes M., Willburger N., Der Soldat und die Götter. Römische Religion am Limes, Stuttgart 2004.

Kemmers F., Coins for a Legion. An Analysis of the Coin Finds from Augustan Legionary Fortress and Flavian canabae legionis at Nijmegen, Mainz 2006.

Koepfer Chr., Himmler F.W., Löffl J. (Hg.), Die römische Armee im Experiment, Berlin 2011.

Konrad M., Römisches Militär in den Orientprovinzen: Defensivmaßnahme oder politisches Instrument?, [in:] K.-S. Freyberger (Hg.), Kulturkonflikte im Vorderen Orient an der Wende vom Hellenismus zur römischen Kaiserzeit, Rahden, Westf. 2003.

Konrad M., Witschel Chr. (Hg.), Römische Legionslager in den Rhein- und Donauprovinzen: Nuclei spätantik-frühmittelalterlichen Lebens?, München 2011.

Kühlborn J.S. (Hg.), Rom auf dem Weg nach Germanien. Geostrategie, Vormarschtrassen und Logistik. Internationales Kolloquium in Delbrück-Anreppen vom 4. bis 6. November 2004, Mainz 2008.

Künzl E., Unter den goldenen Adlern. Der Waffenschmuck des römischen Imperiums, Regensburg-Mainz 2008.

Krause O., Der Arzt und sein Instrumentarium in der römischen Legion, Provinzialrömische Studien 3, Remshalden 2009.

Le Bohec Y., Wolff, C. (Hg.), Les légions de Rome sous le Haut-Empire. Actes du congrès de Lyon (17-19 Septembre 1998), Paris 2000. 
Le Bohec Y., César chef de guerre. César stratège et tacticien. Stratégie et tactique de la république romaine, Paris 2001.

Le Bohec Y., Wolff, C. (Hg.), L'armée romaine de Dioclétien à Valentinien ${ }^{\text {er }}$. Actes du congrès de Lyon (12-14 septembre 2002), Paris 2004.

Le Bohec Y., L'armée romaine dans la tourmente. Une nouvelle approche de la «crise du III siècle», Paris 2009.

Le Bohec Y., Das Römische Heer in der Späten Kaiserzeit. Aus dem Franz. von A. und G. Kolde, Stuttgart 2010.

Le Bohec Y., Alésia. Fin août-début octobre 52 avant J.-C., Paris 2012

Le Bohec Y., La guerre romaine. 58 avant J.-C.-235 après J.-C., Paris 2014.

Le Bohec Y. u.a. (Hg.), The Encyclopedia of the Roman Army, 1-3, Chichester u.a. 2015.

Le Bohec Y., Histoire des guerres romaines. Milieu du VIII e siècle avant J.-C. - 410 après J.-C., Paris 2017.

Lendon J.E., Soldiers and Ghosts. A History of Battle in Classical Antiquity, New Haven 2005.

Levithan J.C., Roman Siege Warfare, Ann Arbor, Mich. 2013.

Liebs D., Bellum iustum in Theorie und Praxis, [in:] M. Avenarius, R. Meyer-Pritzl, C. Möller (Hg.), Ars iuris. Festschrift für Okko Behrends zum 70. Geburtstag, Göttingen 2009, S. 305-318.

Malone St.J., Legio XX Valeria Victrix. Prosopography, Archaeology and History, BAR International Series 1491, Oxford 2006.

Mandl G., Steffenbauer, I. (Hg.), Krieg in der antiken Welt, Essen 2007.

Mann Chr., Militär und Kriegführung in der Antike, Enzyklopädie der griechisch-römischen Antike 9, München 2013

Master J., Provincial Soldiers and Imperial Instability in the Histories of Tacitus, Ann Arbor, Mich. 2016.

Matei-Popescu F., The Roman Army in Moesia inferior, Bukarest 2010.

Matijević K., Transport von Religion durch Soldaten in Obergermanien am Beispiel der Ortsfremden in Mainz/Mogontiacum und Umgebung, Studia Antiqua et Archaeologica 15, 2009, S. 71-144.

Matyszak P., Legionary: The Roman Soldier's (Unofficial) Manual, London 2009.

Meyer-Hermann J., Testamentum militis - das römische Recht des Soldatentestaments. Entwicklung von den Anfängen bis zu Justinian, Aachen 2012.

Nosch M.-L. (Hg.), Wearing the Cloak. Dressing the Soldier in Roman Times, Oxford u.a. 2012.

Östenberg I., Staging the World. Spoils, Captives, and Representations in the Roman Triumphal Procession, Oxford 2009.

Pöppelmann H., Deppmeyer K., Steinmetz W.-D. (Hg.), Roms vergessener Feldzug. Die Schlacht am Harzhorn, Veröffentlichungen des Braunschweigischen Landesmuseums 115, Darmstadt 2013.

Palme B. (Hg.), Die Legionäre des Kaisers. Soldatenleben im römischen Ägypten, Nilus, Studien zur Kultur Ägyptens und des Vorderen Orients 18, Wien 2011.

Parent T.D., The Role and Significance of the Tribal Culture and Warrior Traditions amongst Germanic, Thracian and Mauretanian Soldiers, Diss. University of Hawaii at Manoa 2009. (non vidi)

Petrovitsch H., Legio II Italica, Forschungen in Lauriacum 13, Linz 2006.

Pfahl S.F., Rangabzeichen im römischen Heer der Kaiserzeit, Düsseldorf 2012.

Pferdehirt B. (Hg.), Bürgerrecht und Krise. Die Constitutio Antoniniana 212 n. Chr. und ihre innenpolitischen Folgen. Begleitbuch zur Ausstellung im Römisch-Germanischen Zentralmuseum 20. September 2012 bis 1. Januar 2013, Mainz 2012.

Phang S.E., The Marriage of Roman Soldiers (13 B.C.-235 A.D.). Law and Familiy in the Imperial Army, Columbia Studies in Classical Tradition 24, Leiden 2001. 
Phang S.E., Roman Military Service. Ideologies of Discipline in the Late Republic and Early Principate, Cambridge, Mass. 2008.

Pollard N., Berry J., The Complete Roman Legions, London 2012. Deutsche Ausgabe: Pollard N., Berry, J., Die Legionen Roms. Aus dem Englischen übersetzt von C. Hartz, 3. Aufl., Darmstadt 2016.

Probst P., Eigene Stube, WG oder Mehrbettbelegung? Überlegungen zur Unterbringung von principales im römischen Militärlagern, [in:] S. Panzram, W. Riess, Chr. Schäfer (Hg.), Menschen und Orte der Antike, Festschrift für Helmut Halfmann zum 65. Geburtstag, Pharos, Studien zur griechische-römischen Antike 34, Rahden/Westf. 2015, S. 303-311.

Reuter M., Legio XXX Ulpia Victrix. Ihre Geschichte, ihre Soldaten, ihre Denkmäler, Darmstadt-Mainz 2012.

Richier O., Centuriones ad Rhenum. Les centurions légionnaires des armées romaines du Rhin, Gallia Romana 6, Paris 2004.

Rodgers N., The Roman Army. Legions, Wars and Campaigns. A Military History of the World's first Superpower from the Rise of the Republic and the Might of the Empire to the Fall of the West, London 2005.

Rellstab D.H. (Hg.), Representations of War, Migration, and Refugeehood, New York u.a. 2015.

Roth J.P., Roman Warfare. Cambridge Introduction to Roman Civilization, Cambridge 2009.

Saal P., Adel und Kavallerie. Die Verbindung zwischen Eliten und Militär während der Römischen Republik, Hamburg 2012.

Sage M.M., The Republican Roman Army. A Sourcebook, New York u.a. 2008.

Schmetterer Chr., Die rechtliche Stellung römischer Soldaten im Prinzipat, Philippika, Marburger altertumskundliche Abhandlungen 54, Wiesbaden 2012.

Schalles H.-J., Busch A.W. (Hg.), Waffen in Aktion. Akten der 16. Internationalen Roman Military Equipment Conference, Xanten 13.-16. Juni 2007, Xantener Berichte 16, Mainz 2009.

Schalles H.-J. (Hg.), Die frühkaiserzeitliche manuballista aus Xanten-Wardt, Xantener Berichte 18, Mainz 2010.

Sherman N., Stoic Warriors: The Ancient Philosophy behind the Military Mind, New YorkOxford 2007.

Speidel M.A., Heer und Herrschaft im Römischen Reich der Hohen Kaiserzeit, Stuttgart 2009.

Speidel M.A., Römische Soldaten auf Abwegen. Amtsmissbrauch, Korruption und Fahnenflucht, [in:] M. Reuter, R. Schiavone, Gefährliches Pflaster. Kriminalität im Römischen Reich, Mainz 2011.

Stauner K., Das offizielle Schriftwesen des römischen Heeres von Augustus bis Gallienus (27 v. Chr.-268 n. Chr.). Eine Untersuchung zu Struktur, Funktion und Bedeutung der offiziellen militärischen Verwaltungsdokumentation und zu deren Schreibern, Bonn 2004.

Stäcker J., Princeps und miles. Studien zum Bindungs- und Nahverhältnis von Kaiser und Soldat im 1. und 2. Jahrhundert n. Chr., Spudasmata 91, Hildesheim 2003.

Stallibrass S. (Hg.), Feeding the Roman Army. The Archaeology of Production and Supply in NW Europe, Oxford 2008.

Stoll O., Ehrenwerte Männer. Veteranen im römischen Nahen Osten der Kaiserzeit. Eine Studie zur Wirtschafts-, Sozial- und Kulturgeschichte der nahöstlichen Provinzen anhand papyrologischer und epigraphischer Zeugnisse, Berlin 2015.

Sumner G., Roman Military Clothing. 1: 100 BC-AD 200, Oxford 2002 (Nachdruck 2004); 2: AD 200-400, Oxford 2003

Töpfer K.M., Signa Militaria. Die römischen Feldzeichen in der Republik und im Prinzipat, Monographien des Römisch-Germanischen Zentralmuseums 91, Mainz 2011.

Turner B., Military Defeats, Casualties of War and the Success of Rome. (Dissertation), Chapel Hill 2010.

Ubl H., Waffen und Uniform des römischen Heeres der Prinzipatsepoche nach den Grabreliefs Noricums und Pannoniens, Austria Antiqua 3, Wien 2013 (Diss. Wien 1969). 
Van Lommell K., The Terminology of Medical Discharge and an Identity Shift among the Roman Disabled Veterans, Ancient History Bulletin 27, 2013, S. 65-74.

Volken M., The Water Bag of Roman Soldiers, JRA 21, 2008, S. 264-274.

Weiß P., Militärdiplome für Moesia (Moesia, Moesia superior, Moesia inferior), Chiron 38, 2008, S. 267-316.

Weiß P., Eine honesta missio im Sonderformat. Neuartige Bronzeurkunden für Veteranen der Legionen in Germania superior unter Gordian III., Chiron 45, 2015, S. 23-75.

Weiß P., Konstitutionen eines toten Kaisers. Militärdiplome von Commodus aus dem Jahr 193 n. Chr., [in:] S. Panzram, W. Riess, Chr. Schäfer (Hg.), Menschen und Orte der Antike. Festschrift für Helmut Halfmann zum 65. Geburtstag, Pharos, Studien zur griechischrömischen Antike 34, Rahden/Westf. 2015, S. 273-280.

Wilkes J.J. (Hg.), Documenting the Roman Army. Essays in Honour of Margaret Roxan, London 2003.

Wolff C., Déserteurs et transfuges dans l'armée romaine à l'époque républicaine, Neapel 2009.

Wolff C. (Hg.), L'armée romaine et la religion sous le Haut-Empire romain. Actes du quatrième Congrès de Lyon (26-28 octobre 2006), Paris 2009.

Wolff C. (Hg.), Le métier de soldat dans le monde romain. Actes du cinquième Congrès de Lyon (23-25 septembre 2010), Paris 2012.

Wooliscroft D.J., Roman Military Signalling, Stroud 2001.

Zahariade M., The Thracians in the Roman Imperial Army. From the First to the Third Century A.D., Cluj-Napoca 2009.

Zehetner St., Der Signifer. Stellung und Aufgaben in der kaiserzeitlichen Armee, Saarbrücken 2011. 